\title{
Joint Power Allocation and Precoding for Network Coding-Based Cooperative Multicast Systems
}

\author{
Jun Li and Wen Chen, Member, IEEE
}

\begin{abstract}
In this letter, we propose two power allocation schemes based on the statistical channel state information (CSI) and instantaneous $s \rightarrow r$ CSI at transmitters, respectively, for a $2-N-2$ cooperative multicast system with nonregenerative network coding. Then the isolated precoder and the distributed precoder are, respectively, applied to the schemes to further improve the system performance by achieving the full diversity gain. Finally, we demonstrate that joint instantaneous $s \rightarrow r$ CSI-based power allocation and distributed precoder design achieve the best performance.
\end{abstract}

Index Terms-Cooperative multicast network, frame error probability, network coding, power allocation, precoder design.

\section{INTRODUCTION}

$\mathbf{N}$ ETWORK coding has been proved to achieve the network multicast capacity bound in the wireline systems [1]. Recently, how to leverage network coding in wireless physical layer networks for system capacity improvement has drawn increasing interest [2]-[4]. However, these works are based on the multiaccess relay channels model and unicast model.

In this letter, we study the multicast model with two sources, two destinations, and $N$ relays ( $2-N-2$ system) by following the second scheduling strategy in [5], where relays are arranged in the round-robin way. We suppose that $s_{1}$ as well as $s_{2}$ broadcast their information to the two destinations $d_{1}$ and $d_{2}$ simultaneously. From Fig. 1, we can see $d_{1}$ (or $d_{2}$ ) is out of the transmission range of $s_{2}$ (or $s_{1}$ ). The shared relays can help $s_{1}$ (or $s_{2}$ ) reach their destinations. By the wireless network coding method, there are two time slots:

1) $s_{1} \rightarrow\left\{r, d_{1}\right\}$ with $X_{s_{1}} ; s_{2} \rightarrow\left\{r, d_{2}\right\}$ with $X_{s_{2}}$;

2) $r \rightarrow\left\{d_{1}, d_{2}\right\}$ with $f\left(X_{s_{1}}, X_{s_{2}}\right)$

where $f(\cdot)$ is certain mapping mechanism. We focus on the nonregenerative network coding where mixed signals are scaled and retransmitted to the destinations without decoding at relays. We propose two power allocation schemes based on the statistical channel state information (CSI) and the instantaneous $s \rightarrow r$ CSI at sources, respectively. The isolated precoders and distributed precoders are, respectively, jointly working with the schemes to achieve the full diversity gain. We will demonstrate

Manuscript received June 24, 2008; revised August 11, 2008. This work was supported in part by NSF China \#60672067, in part by NSF Shanghai \#06ZR14041, in part by Shanghai-Canada NRC \#06SN07112, in part by Cultivation Fund of the Key Scientific and Technical Innovation Project, Ministry of Education of China \#706022, in part by Program for New Century Excellent Talents in University \#NCET-06-0386, in part by PUJIANG Talents \#07PJ4046, and in part by Huawei Fund for Sciences and Technologies in Universities \#YJCB2008048WL. The associate editor coordinating the review of this manuscript and approving it for publication was Dr. Nikolaos V. Boulgouris.

The authors are with the Department of Electronic Engineering, Shanghai Jiaotong University, Shanghai, 200240, China (e-mail: jleesr80@sjtu.edu.cn; wenchen@sjtu.edu.cn).

Digital Object Identifier 10.1109/LSP.2008.2006330

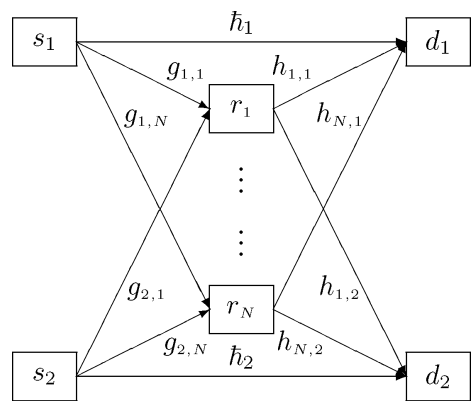

Fig. 1. Multicast cooperative channels with $N$ relays.

that the joint instantaneous $s \rightarrow r$ CSI-based power allocation and distributed precoder can achieve the best performance against the other combinations in the terms of system frame error probability (SFEP).

\section{SYSTEM MODEL}

Channel coefficients are shown in Fig. 1 with zero means and unit variances. The noise variances are equal to $\sigma^{2}$ in all the receivers. To normalize the power, we denote $P$ as the average total network transmission power over a time slot. Then we define the system SNR as $\rho \triangleq P / \sigma^{2}$. We define $\mathbf{x}_{s} \triangleq\left[x_{s_{1}, 1}, x_{s_{2}, 1}, x_{s_{1}, 2}, x_{s_{2}, 2}, \ldots, x_{s_{1}, N}, x_{s_{2}, N}\right]^{T}$ as a system frame which is composed of symbols from two sources. All symbols are equally probable from the QAM constellation set with zero means and variances $2 P$. The symbol vector in the system frame for $s_{k}(k=1,2)$ is denoted as $\mathbf{x}_{s_{k}}=\left[x_{s_{k}, 1}, x_{s_{k}, 2}, \ldots, x_{s_{k}, N}\right]^{T}$. Since each relay is only used once during a frame period, we denote the signal received by the $i$ th relay as $y_{r_{i}}$. The symbol vector received by the $k$ th destination is denoted as $\mathbf{y}_{d_{k}}=\left[y_{d_{k}, 1}, y_{d_{k}, 2}, \ldots, y_{d_{k}, 2 N-1}, y_{d_{k}, 2 N}\right]^{T}$.

The total power consumed during a frame period is

$$
\mathcal{E}\left\{\mathbf{k}_{1}^{T}\left|\mathbf{x}_{s_{1}}\right|^{2}+\mathbf{k}_{2}^{T}\left|\mathbf{x}_{s_{2}}\right|^{2}+\left|\mathbf{b}^{T} \mathbf{y}_{r}\right|^{2}\right\}=2 N P
$$

where $\mathbf{y}_{r}=\left[y_{r_{1}}, y_{r_{2}}, \ldots, y_{r_{N}}\right]^{T}$. The power is distributed according to the power allocation factor vectors, i.e., $\mathbf{k}_{k}=\left[\kappa_{k, 1}, \ldots, \kappa_{k, N}\right]^{T}$ is the factor vector for the $k$ th source and $\mathbf{b}=\left[b_{1}, \ldots, b_{N}\right]^{T}$ is the amplification factor vector where $b_{i}=\sqrt{2 \tau_{i} P /\left(2 \kappa_{1, i} P+2 \kappa_{2, i} P+\sigma^{2}\right)}$ is the factor for the $i$ th relay. Note that $\tau_{i}$ is the corresponding power allocation factor for the $i$ th relay. We denote $\mathbf{t}=\left[\tau_{1}, \ldots, \tau_{N}\right]^{T}$.

The received signals of the $k$ th destination for the $(2 i-1)$ th and $2 i$ th time slots are, respectively, written as

$$
\begin{aligned}
y_{d_{k}, 2 i-1} & =\hbar_{k} \sqrt{\kappa_{k, i}} x_{s_{k}, i}+v_{d_{k}, 2 i-1} \\
y_{d_{k}, 2 i} & =b_{i} h_{i, k}\left(g_{1, i} \sqrt{\kappa_{1, i}} x_{s_{1}, i}+g_{2, i} \sqrt{\kappa_{2, i}} x_{s_{2}, i}+v_{r_{i}}\right)+v_{d_{k}, 2 i} .
\end{aligned}
$$


Note that $v_{r_{i}}$ is the noise observed by the $i$ th relay and $v_{d_{k}, 2 i}$ is the noise observed by $d_{k}$ in the $2 i$ th time slot. Joint ML decoding is performed at the end of each frame period.

\section{Performance Analysis And Improvements}

We measure the performance by system frame error probability (SFEP). We define that a frame is successfully transmitted if and only if both destinations can successfully receive the frame. So the SFEP of the multicast system is

$$
P_{s y s}=P_{d_{k}}\left(1-P_{d_{\bar{k}}}\right)+P_{d_{\bar{k}}}\left(1-P_{d_{k}}\right)+P_{d_{k}} P_{d_{\bar{k}}}
$$

where $\bar{k}$ is the complementary element of $k$ in set $\{1,2\}$, and $P_{d_{k}}$ is the FEP of $d_{k}$. We improve the performance by combining power allocation and precoders according to the knowledge of the CSI at sources.

\section{A. Statistical CSI-Based Power Allocation}

Due to the symmetrical quality of $P_{\text {sys }}$ and according to the fact that each channel variance is equal, power should be equally allocated to each symbol of the two sources, i.e., $\mathbf{k}_{1}=\mathbf{k}_{2}=$ $[\kappa, \ldots, \kappa]^{T}$ and $\mathbf{t}=[\tau, \ldots, \tau]^{T}$. Then the amplification factor $b=\sqrt{2 \tau P /\left(4 \kappa P+\sigma^{2}\right)}$. Then we get the statistical CSI-based power allocation scheme as follows.

Theorem 1: When $\rho$ is large enough, the statistical CSI-based optimal power allocation scheme chooses the power allocation factors as $\kappa=1 / 2(N+1)$ and $\tau=N /(N+1)$.

Proof: On the condition that only statistical CSI is available, $P_{d_{k}}$ can be deduced by the average pairwise error probability (PEP). Note that FEP $=2^{2 R N}$ PEP since there are total $2^{2 R N}$ codewords, where $R$ is the transmission rate. So to find out the optimal relation between $\kappa$ and $\tau$, we first come to the average PEP of the destinations. We rewrite (2) in matrix form as $\mathbf{x}_{d_{k}}=\mathbf{X}_{2 N} \mathbf{h}_{2 N}+\mathbf{v}_{2 N}$ and get the PEP by developing the method in [7]

$P_{P E, d_{k}}=\frac{1}{\pi} \int_{0}^{\pi / 2} \mathcal{E}_{\mathbf{h}_{2 N}}\left\{\exp \left(-\rho \frac{\mathbf{h}_{2 N}^{H} \mathbf{U}_{2 N}^{H} \boldsymbol{\Sigma}_{\mathbf{v}}^{-1} \mathbf{U}_{2 N} \mathbf{h}_{2 N}}{8 \sin ^{2} \theta}\right)\right\} \mathrm{d} \theta$

where $\mathbf{U}_{2 N}=\mathbf{X}_{2 N}-\hat{\mathbf{X}}_{2 N}$ is the decoding error matrix and $\boldsymbol{\Sigma}_{\mathbf{v}}$ is the variance matrix of $\mathbf{v}_{2 N} \cdot \mathbf{h}_{2 N}$ can be written by $\mathbf{T}_{2 N} \mathbf{g}_{2 N}$ where $\mathbf{g}_{2 N}=\left[\hbar_{k}, g_{1,1}, g_{2,1}, g_{1,2}, g_{2,2}, \ldots, g_{1, N}, g_{2, N}\right]^{T}$ and $\mathbf{T}_{2 N}=\operatorname{diag}\left(1, b h_{1, k}, b h_{1, k}, b h_{2, k}, b h_{2, k}, \ldots, b h_{N, k}, b h_{N, k}\right)$. Note that for a random column vector $\mathbf{z} \sim \mathcal{N}\left(0, \boldsymbol{\Sigma}_{\mathbf{z}}\right)$ and a Hermitian matrix $\mathbf{H}$, there is $\mathcal{E}\left[\exp \left(-\mathbf{z}^{H} \mathbf{H z}\right)\right]=1 / \operatorname{det}(\mathbf{I}+$ $\left.\boldsymbol{\Sigma}_{\mathbf{z}} \mathbf{H}\right)$. We take expectation with respect to $\mathbf{g}_{2 N}$ and let $\mathbf{y}=$ $\left[y_{1}, y_{2}, \ldots, y_{N}\right]$ where $y_{i}=\left|h_{i, k}\right|^{2}$ with the probability distribution function $e^{-y_{i}}$. Then the PEP is written as

$$
P_{P E, d_{k}}=\frac{1}{\pi} \int_{0}^{\pi / 2} \int_{0}^{\infty} \cdots \int_{0}^{\infty} \frac{\exp \left(-\sum_{j=1}^{N} y_{j}\right)}{\operatorname{det}(\mathbf{A})} \mathrm{d} \mathbf{y} \mathrm{d} \theta .
$$

In (5), $\operatorname{det}(\mathbf{A})=\operatorname{det}\left(\mathbf{A}_{0}\right) \operatorname{det}\left(\mathbf{A}_{1}\right) \cdots \operatorname{det}\left(\mathbf{A}_{N}\right)$, where

$$
\begin{aligned}
\mathbf{A}_{0} & =1+\frac{\rho}{8 \sin ^{2} \theta} \sum_{j=1}^{N}\left|u_{s_{k}, j}\right|^{2} \\
\mathbf{A}_{i} & =\left(\begin{array}{cc}
1+\lambda_{i}\left|u_{s_{1}, i}\right|^{2} & \lambda_{i} u_{s_{1}, i}^{*} u_{s_{2}, i} \\
\lambda_{i} u_{s_{1}, i} u_{s_{2}, i}^{*} & 1+\lambda_{i}\left|u_{s_{2}, i}\right|^{2}
\end{array}\right)
\end{aligned}
$$

$u_{s_{k}, j}=\sqrt{\kappa / P}\left(x_{s_{k}, j}-\hat{x}_{s_{k}, j}\right)$ is the decoding error value of the $j$ th symbol, and $\lambda_{i}=b^{2}\left|h_{i, k}\right|^{2} \rho / 8 \sin ^{2} \theta\left(1+b^{2}\left|h_{i, k}\right|^{2}\right)$. So $\operatorname{det}\left(\mathbf{A}_{i}\right)=1+\lambda_{i}\left|u_{s_{1}, i}\right|^{2}+\lambda_{i}\left|u_{s_{2}, i}\right|^{2}$. Then we turn to the integral for $\int_{0}^{\infty}\left(e^{-y_{i}} / \operatorname{det}\left(\mathbf{A}_{i}\right)\right) \mathrm{d} y_{i}$, i.e.,

$$
\begin{gathered}
\int_{0}^{\infty} \frac{e^{-y_{i}}}{1+\frac{b^{2} y_{i} \rho\left|u_{s_{1}, i}\right|^{2}}{8 \sin ^{2} \theta\left(1+b^{2} y_{i}\right)}+\frac{b^{2} y_{i} \rho\left|u_{s_{2}, i}\right|^{2}}{8 \sin ^{2} \theta\left(1+b^{2} y_{i}\right)}} \mathrm{d} y_{i} \\
\quad=\int_{0}^{\infty} \frac{b^{2}}{\gamma_{i}}\left(1+\frac{\frac{1}{b^{2}}-\frac{1}{\gamma_{i}}}{y_{i}+\frac{1}{\gamma_{i}}}\right) e^{-y_{i}} \mathrm{~d} y_{i} \\
=\frac{b^{2}}{\gamma_{i}}\left(1+\left(\frac{1}{b^{2}}-\frac{1}{\gamma_{i}}\right) J\left(\frac{1}{\gamma_{i}}\right)\right)
\end{gathered}
$$

where $\gamma_{i}=b^{2}+\alpha_{i}+\beta_{i}, \alpha_{i}=\rho b^{2}\left|u_{s_{1}, i}\right|^{2} / 8 \sin ^{2} \theta$, and $\beta_{i}=$ $\rho b^{2}\left|u_{s_{2}, i}\right|^{2} / 8 \sin ^{2} \theta$. From [8], function $J(\nu)$ can be expressed as

$$
J(\nu)=\int_{0}^{\infty} \frac{e^{-\mu}}{\mu+\nu} \mathrm{d} \mu=-e^{\nu}\left(\varphi+\ln \nu+\sum_{j=1}^{\infty}\left(\frac{(-1)^{j} \nu^{j}}{(j ! j)}\right)\right)
$$

where $\varphi$ is the Euler constant. Then we assume $\rho$ is large enough to work out the asymptotic solution. If $\nu=c_{1} \rho^{-1}+O\left(c_{2} \rho^{-2}\right)$ $\left(0<c_{1}, c_{2}<\infty\right)$, when $\rho$ is large enough

$$
J(\nu)=\ln \rho+O\left(\left|\ln c_{1}\right|\right) .
$$

Meanwhile, we have

$$
\frac{1}{\gamma_{i}}=\frac{1}{b^{2}} \frac{8 \sin ^{2} \theta \rho^{-1}}{\left|u_{s_{1}, i}\right|^{2}+\left|u_{s_{2}, i}\right|^{2}}+O\left(\frac{\rho^{-2}}{\left|u_{s_{1}, i}\right|^{2}+\left|u_{s_{2}, i}\right|^{2}}\right) .
$$

Then

$$
\begin{aligned}
& \frac{b^{2}}{\gamma_{i}}\left(1+\left(\frac{1}{b^{2}}-\frac{1}{\gamma_{i}}\right) J\left(\frac{1}{\gamma_{i}}\right)\right) \\
& =\frac{8 \sin ^{2} \theta b^{-2} \rho^{-1}}{\left|u_{s_{1}, i}\right|^{2}+\left|u_{s_{2}, i}\right|^{2}}\left(\ln \rho+O\left(\left|\ln \left(\left|u_{s_{1}, i}\right|^{2}+\left|u_{s_{2}, i}\right|^{2}\right)\right|\right)\right) .
\end{aligned}
$$

On the other hand, we denote $K=\int_{0}^{\pi / 2} \sin ^{2(N+1)} \theta \mathrm{d} \theta$. When $\rho$ is large enough, we can write

$$
P_{P E, d_{k}}=\frac{K b^{-2 N} \rho^{-(N+1)} \ln ^{N} \rho}{\sum_{j=1}^{N}\left|u_{s_{k}, j}\right|^{2} \prod_{j=1}^{N}\left(\left|u_{s_{k}, j}\right|^{2}+\left|u_{s_{\bar{k}}, j}\right|^{2}\right)} .
$$

Since $\mathcal{E}\left(\left|u_{s_{k}, i}\right|^{2}\right)=\mathcal{E}\left(\left|x_{s_{k}, i}-\hat{x}_{s_{k}, i}\right|^{2}\right)=4 \kappa$

$$
\mathcal{E}\left(P_{s y s}\right) \sim \frac{\rho^{-(N+1)} \ln ^{N} \rho}{\kappa \tau^{N}} .
$$

To minimize $\mathcal{E}\left(P_{\text {sys }}\right)$, we should enlarge the value of $\kappa \tau^{N}$. So the power allocation factors is worked out by maximizing $\kappa \tau^{N}$ subject to the power constraint $2 \kappa+\tau=1$.

\section{B. Instantaneous $s \rightarrow r$ CSI-Based Power Allocation}

Since the coefficients of $s \rightarrow r$ link should be notified to destinations by relays for decoding, it is rational to assume that the instantaneous $s \rightarrow r$ CSI can also be obtained by sources without extra overheads. In this case, power is only reallocated 
between the two sources while remains unchanged for the relays. Then we get the instantaneous $s \rightarrow r$ CSI-based power allocation scheme by water filling [10] as follows.

Theorem 2: The instantaneous $s \rightarrow r$ CSI-based optimal power allocation scheme is

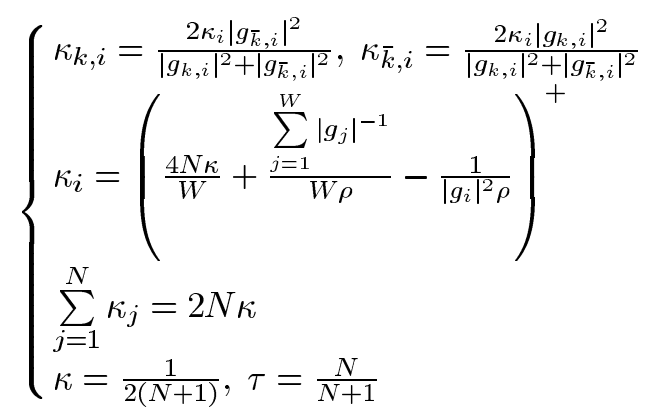

where $\left|g_{i}\right|^{2}=\left|g_{k, i} g_{\bar{k}, i}\right|^{2} /\left(\left|g_{k, i}\right|^{2}+\left|g_{\bar{k}, i}\right|^{2}\right), \kappa_{s_{k}, i}$ is the power allocation factor of the $k$ th source when transmitting to the $i$ th relay, and $W$ is the number of positive $\kappa_{i}$. Moreover, instantaneous $s \rightarrow r$ CSI is also used to pre-equalize the channels phase which produces a coherent superposition of signals from two sources which can achieve better performance.

Proof: When $s \rightarrow r$ CSI is available at sources, power is only reallocated between two sources. So the $\kappa$ and $\tau$ is unchanged. In the $(2 i-1)$ th time slot, the $i$ th relay receives the mixed signals from the two sources which can be seen as a multiaccess model. We then turn to the achievable capacity region of the channel between each source and the $i$ th relay when joint ML decoding is performed. As well known, the mutual information between the sources and relay on the channel realization $\boldsymbol{g}_{i}$ is

$$
I\left(s_{k}, s_{\bar{k}} ; r_{i} \mid \boldsymbol{g}_{i}\right)=\frac{1}{2} \log \left(1+2\left(\left|g_{k, i}\right|^{2} \kappa_{k, i}+\left|g_{\bar{k}, i}\right|^{2} \kappa_{\bar{k}, i}\right) \rho\right) .
$$

According to [9], we suppose that each source splits its power into the same pieces, i.e., $2 \kappa_{k, i} \rho=M \triangle \rho_{k}$ for the $k$ th source. Two sources alternatively pour one piece of their power into the channels to gain the rate growth $\triangle R\left(s_{k}^{m}\right)$ in the $m$ th round. Let $\triangle \rho_{k} \rightarrow 0$, and then

$$
\triangle R\left(s_{k}^{m}\right)=\frac{1}{2}\left|g_{k, i}\right|^{2} \triangle \rho_{k} \eta_{i, m}, \triangle R\left(s_{\bar{k}}^{m}\right)=\frac{1}{2}\left|g_{\bar{k}, i}\right|^{2} \triangle \rho_{\bar{k}} \eta_{i, m}
$$

where

$$
\eta_{i, m}=\frac{1}{1+m \sum_{j=1}^{2}\left|g_{j, i}\right|^{2} \triangle \rho_{j}}
$$

Then the achievable capacity of the channel between the $k$ th source and the $i$ th relay is

$$
I\left(s_{k} ; r_{i} \mid \boldsymbol{g}_{i}\right)=\int_{0}^{2 \kappa_{k, i} \rho} \frac{\frac{1}{2}\left|g_{k, i}\right|^{2}}{1+m \sum_{j=1}^{2}\left|g_{j, i}\right|^{2} \triangle \rho_{j}} \mathrm{~d} \rho_{k}
$$

since in joint ML decoding, $\kappa_{k, i}$ and $\kappa_{\bar{k}, i}$ are already known. So in (19), by replacing $\triangle \rho_{\bar{k}}$ with $\left(\kappa_{\bar{k}, i} / \kappa_{k, i}\right) \triangle \rho_{k}$

$$
I\left(s_{k} ; r_{i} \mid g_{i}\right)=\int_{0}^{2 \kappa_{k, i} \rho} \frac{\frac{1}{2}\left|g_{k, i}\right|^{2} \mathrm{~d} \rho_{k}}{1+\left(\left|g_{k, i}\right|^{2}+\frac{\kappa_{\bar{k}, i}}{\kappa_{k, i}}\left|g_{\bar{k}, i}\right|^{2}\right) \rho_{k}} .
$$

Thus, we get

$$
I\left(s_{k} ; r_{i} \mid \boldsymbol{g}_{i}\right)=\frac{\kappa_{k, i}\left|g_{k, i}\right|^{2}}{\kappa_{k, i}\left|g_{k, i}\right|^{2}+\kappa_{\bar{k}, i}\left|g_{\bar{k}, i}\right|^{2}} I\left(s_{k}, s_{\bar{k}} ; r_{i} \mid \boldsymbol{g}_{i}\right) .
$$

Then power allocation scheme for the two sources in the $(2 i-$ 1)th time slot, namely, local power allocation when $s \rightarrow r$ CSI is available, is to make

$$
\max _{\kappa_{k, i}, \kappa_{\bar{k}, i}} \min \left\{I\left(s_{k} ; r_{i} \mid \boldsymbol{g}_{i}\right), I\left(s_{\bar{k}} ; r_{i} \mid \boldsymbol{g}_{i}\right)\right\} .
$$

Due to the symmetrical quality of the channel model, we should keep the balance of the system, i.e., $I\left(s_{k} ; r_{i} \mid \boldsymbol{g}_{i}\right)=I\left(s_{\bar{k}} ; r_{i} \mid \boldsymbol{g}_{i}\right)$. Then

$$
\kappa_{k, i}\left|g_{k, i}\right|^{2}=\kappa_{\bar{k}, i}\left|g_{\bar{k}, i}\right|^{2}, \kappa_{k, i}+\kappa_{\bar{k}, i}=2 \kappa .
$$

So we get the local power allocation scheme as

$$
\kappa_{k, i}=\frac{2 \kappa\left|g_{\bar{k}, i}\right|^{2}}{\left|g_{k, i}\right|^{2}+\left|g_{\bar{k}, i}\right|^{2}}, \kappa_{\bar{k}, i}=\frac{2 \kappa\left|g_{k, i}\right|^{2}}{\left|g_{k, i}\right|^{2}+\left|g_{\bar{k}, i}\right|^{2}} .
$$

After local power allocation, the channels from the two sources to the $i$ th relay suffer the same fading $\left|g_{k, i} g_{\bar{k}, i}\right|^{2} /\left(\left|g_{k, i}\right|^{2}+\left|g_{\bar{k}, i}\right|^{2}\right)$. Then a frame period can be divided into $N$ orthogonal time division channels with channel fading $\left|g_{i}\right|^{2}$ for the $i$ th channel. According to [10], we get the global power allocation scheme (14) by water filling scheme.

\section{Precoders Design}

From (12), it is obvious that the system cannot achieve the full diversity gain, i.e., the denominator of (12) has the chance to equal to 0 , which decreases the diversity orders. Precoder is then applied to enhance the diversity gain.

1) Isolated Precoder: If only statistical CSI is available, precoders are designed in the same way for both sources, i.e., isolated precoder. We follow the precoder design in MIMO [6] which achieves full diversity gain. Then for each source, the precoder matrix is

$$
\Theta_{s}=\frac{1}{\sqrt{N}}\left(\begin{array}{cccc}
1 & \alpha_{1} & \cdots & \alpha_{1}^{N-1} \\
\vdots & \vdots & & \vdots \\
1 & \alpha_{N} & \cdots & \alpha_{N}^{N-1}
\end{array}\right)_{N \times N}
$$

where $\left\{\alpha_{i}\right\}_{i=1}^{N}$ have unit modulus. Thus, the transmitted signals for the $k$ th source becomes $\sqrt{\kappa} \boldsymbol{\Theta}_{s} \mathbf{x}_{s_{k}}$.

Remark 1: When $\rho \rightarrow \infty$, by applying statistical CSI-based power allocation scheme and isolated precoders in each source, the average PEP of $d_{k}$ is then

$$
P_{P E, d_{k}}=\frac{(N+1)^{N+1} K \rho^{-(N+1)} \ln ^{N} \rho}{\left(\frac{N}{2}\right)^{N} \sum_{j=1}^{N} \mu_{s_{k}, j} \prod_{j=1}^{N}\left(\mu_{s_{1}, j}+\mu_{s_{2}, j}\right)}
$$

where $\mu_{s_{k}, j}=(1 / N)\left|\sum_{n=1}^{N} \alpha_{i}^{n-1} u_{s_{k}, n}\right|^{2}$.

By applying precoder, the denominator of (25) equals to 0 if and only if the frame can be successfully decoded. Then the whole system can achieve full diversity gain. However, isolated precoders design is not suitable for instantaneous $s \rightarrow r$ CSI-based power allocation scheme, in which it cannot achieve the full diversity gain. More specifically, for the $i$ th 


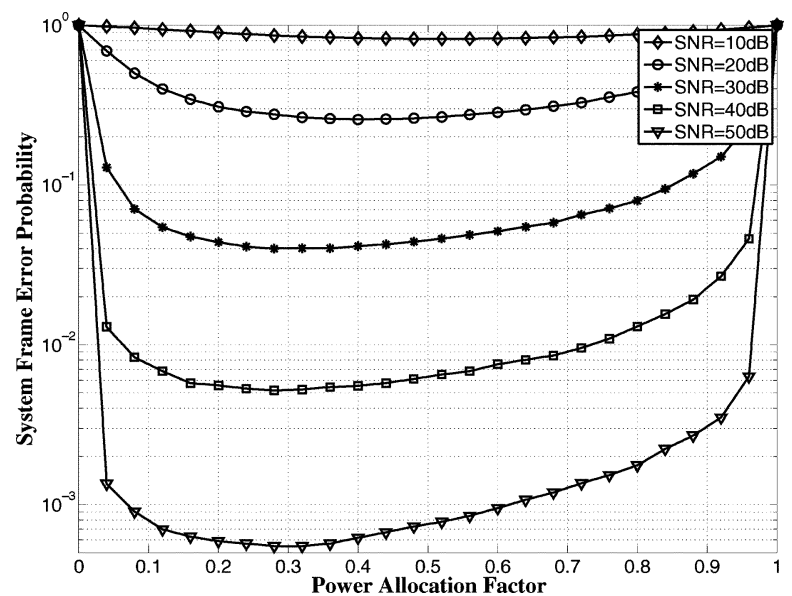

Fig. 2. SFEP in two-relay scenario with different power allocation schemes using 4-QAM modulation. Power allocation factor is the value of $2 \kappa$.

relay, $\mu_{s_{k}, i}+\mu_{s_{k}, i}=(1 / N)\left|\sum_{j=1}^{N} \alpha_{i}^{j-1}\left(u_{s_{k}, j}+u_{s_{k}, j}\right)\right|^{2} \cdot \mathrm{A}$ wrong decoding of the $j$ th symbol $\hat{x}_{s_{k}, j}$ and $\hat{x}_{s_{\bar{k}}, j}$ in the two symbol vectors may cause $u_{s_{k}, j}+u_{s_{\bar{k}}, j}=0$, which lead to zero in the denominator of PEP expression and hence the lower diversity gain. Then we propose the distributed precoder.

2) Distributed Precoder: We first construct a $2 N \times 2 N$ matrix as (24). Then arbitrary $N$ rows are selected to form a new matrix $\boldsymbol{\Theta}$. The precoder matrix $\boldsymbol{\Theta}_{s_{1}}$ for $s_{1}$ comes from the odd columns of the $\Theta$ and the precoder matrix $\Theta_{s_{2}}$ for $s_{2}$ comes from the even columns of the $\Theta$, i.e., for the $k$ th source

$\boldsymbol{\Theta}_{s_{k}}=\frac{1}{\sqrt{N}}\left(\begin{array}{ccccc}\alpha_{1}^{k-1} & \cdots & \alpha_{1}^{2 j+k-1} & \cdots & \alpha_{1}^{2 N+k-3} \\ \vdots & & \vdots & & \vdots \\ \alpha_{N}^{k-1} & \cdots & \alpha_{N}^{2 j+k-1} & \cdots & \alpha_{N}^{2 N+k-3}\end{array}\right)_{N \times N}$

where $j \in\{0, \ldots, N-1\}$. By this mean, signal superposed in the $i$ th relay will be equal to $\left(\kappa_{i}\left|g_{k, i} g_{\bar{k}, i}\right|^{2} /\left(\left|g_{k, i}\right|^{2}+\left|g_{\bar{k}, i}\right|^{2}\right)\right) \boldsymbol{\theta}^{i} \mathbf{x}_{s}$, where $\boldsymbol{\theta}^{i}$ denotes the $i$ th row of $\boldsymbol{\Theta}$. It means that in PEP expression, $\mu_{s_{k}, i}+\mu_{s_{\bar{k}}, i}=(1 / N)\left|\sum_{j=1}^{N} \alpha_{i}^{j-1}\left(u_{s_{k}, j}+\alpha_{i} u_{s_{\bar{k}}, j}\right)\right|^{2}$, which achieves full diversity gain.

\section{NUMERICAL RESULTS}

In our Monte Carlo simulations, we choose 4-QAM modulation with two relays. Each frame has four symbols, and each SFEP value is simulated by $10^{6}$ i.i.d. frames. Fig. 2 shows the SFEP under statistical CSI-based power allocation schemes with different values of power allocation factor $\kappa$. We can see that when $\rho$ is large enough, systems reaches the lowest SFEP for $2 \kappa=1 / 3$ and $\tau=2 / 3$, which validates Theorem 1 .

Fig. 3 compares the SFEP in five cases: 1) statistical CSIbased average power allocation scheme (SAPAS) without precoder (NP); 2) statistical CSI-based optimal power allocations scheme (SOPAS) without precoder; 3) instantaneous $s \rightarrow r$ CSI-based optimal power allocation scheme (IOPAS) with isolated precoders (IP); 4) SOPAS with IP; 5) IOPAS with DP. By SAPAS, both sources and relays consume the same power. We can see that power allocation scheme and precoders can distinctly improve the performance. Since IP with IOPAS cannot achieve full diversity gain, it leads to worse performance even

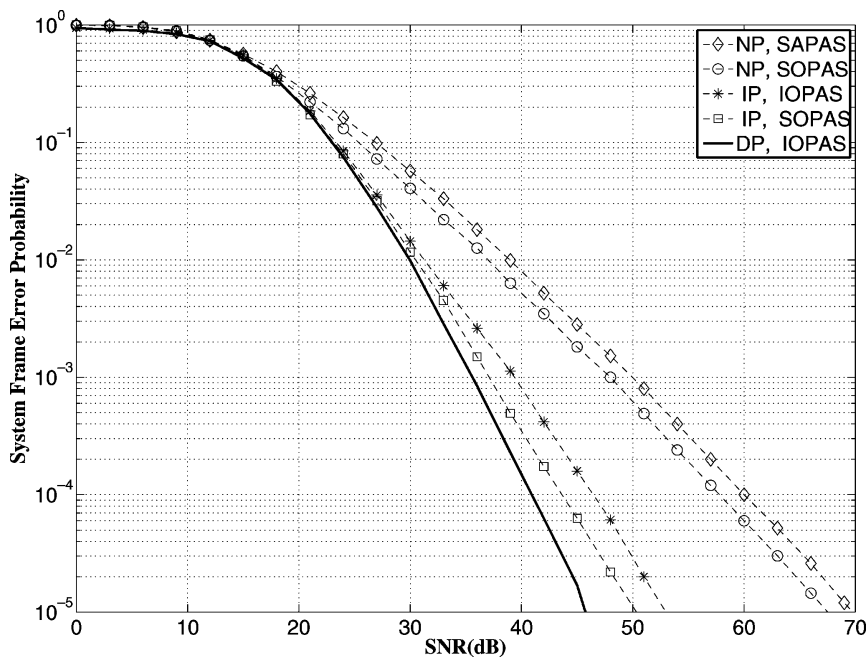

Fig. 3. SFEP in two-relay scenario using 4-QAM modulation in five cases.

than that with SOPAS. However, DP with IOPAS achieves the best performance since it makes the system achieve full diversity with larger Euclidian distance.

\section{CONCLUSION}

In this letter, we propose two power allocation schemes and two precoders for $2-N-2$ multicast systems with nonregenerative network coding. Although IP can help SOPAS achieve full diversity gain, it cannot well help IOPAS. However, DP jointly with IOPAS can make the system not only achieve full diversity gain but also outperform any other combinations of power allocation schemes and precoders in the term of SFEP.

\section{REFERENCES}

[1] R. Ahlswede, N. Cai, S.-Y. R. Li, and R. W. Yeung, "Network information flow," IEEE Trans. Inf. Theory, vol. 46, no. 4, pp. 1204-1216, Jul. 2000.

[2] S. Zhang, S.-C. Liew, and P. P. Lam, "Hot topic: Physical-layer network coding," in Proc. 12th Аnnu. Int. Conf. Mobile Computing and Networking (MobiCom), Los Angeles, CA, Sep. 23-26, 2006, pp. 358-365.

[3] T. Wang and G. B. Giannakis, "High-throughput cooperative communications with complex field network coding," in Proc. 41st Annu. Int. Conf. Information Sciences and Systems (CISS), Baltimore, MD, Mar. 14-16, 2007, pp. 253-258.

[4] P. Popovski and H. Yomo, "Wireless network coding by amplify-andforward for bi-directional traffic flows," IEEE Commun. Letters, vol. 11, no. 1, pp. 16-18, Jan. 2007.

[5] R. U. Nabar, H. Bölcskei, and F. W. Kneubuhler, "Fading relay channels: Performance limits and space-time signal design," IEEE J. Sel. Areas Commun., vol. 22, no. 6, pp. 1099-1109, Aug. 2004.

[6] Y. Xin, Z. Wang, and G. B. Giannakis, "Space-time diversity systems based on linear constellation precoding," IEEE Trans. Wireless Commun., vol. 2, no. 2, pp. 294-309, Mar. 2003.

[7] Y. Ding, J.-K. Zhang, and K. M. Wong, "The amplify-and-forward half-duplex cooperative system: Pairwise error probability and precoder design," IEEE Trans. Signal Process., vol. 55, no. 2, pp. 605-617, Feb. 2007.

[8] N. N. Lebedev, Special Functions and Their Applications. Englewood Cliffs, NJ: Prentice-Hall, 1965.

[9] D. N. C. Tse and S. V. Hanly, "Multiaccess fading channels-Part I : Polymatroid structure, optimal resource allocation and throughput capacities," IEEE Trans. Inf. Theory, vol. 44, no. 7, pp. 2796-2815, Nov. 1998.

[10] G. Caire, G. Taricco, and E. Biglieri, "Optimum power control over fading channels," IEEE Trans. Inf. Theory, vol. 45, no. 5, pp. 1468-1489, Jul. 1998. 